\title{
Heavy Metals and Neurodevelopment of Children in Low and Middle-Income Countries: A Systematic Review
}

\author{
Yi Yan Heng ${ }^{1}$, Iqra Asad ${ }^{2}$, Bailey Coleman², Megan McHenry ${ }^{3}$ \\ ${ }^{1}$ Indiana University School of Medicine; ${ }^{2}$ Indiana University-Purdue University \\ Indianapolis; ${ }^{3}$ Indiana University School of Medicine, Department of Pediatrics
}

Background: The presence of harmful environmental exposures contributes to $>25 \%$ of deaths and diseases worldwide. Child neurodevelopment is negatively impacted by environmental exposures, such as certain heavy metals. In low and middle-income countries, where numerous risk factors for poor neurodevelopment exist, it is unclear if this negative impact persists. Our objective with this systematic review is to evaluate the association between heavy metals and the neurodevelopment of children in low-and-middle income countries.

Methods: We conducted a search on PubMed MEDLINE, Embase, Cochrane Library, CINAHL, PsychInfo, Scopus, and Web of Science for articles that included individuals below the age of 18 , quantitively measured exposure to a heavy metal and used a standardized measurement of neurodevelopment.

Results: Of the 15,250 screened articles, 292 full-text articles were reviewed, and 96 articles met inclusion criteria. These studies were conducted in 18 countries and included 46,080 participants ranging from birth -18 years old. At least one domain of neurodevelopment was inversely correlated with 8/11 of arsenic studies, 3/3 of cadmium studies, 29/32 of lead studies, $11 / 11$ of manganese studies, 9/18 of mercury studies, and 21/21 studies with multiple metals. There was no consistent evidence of arsenic affecting behavior; mercury negatively affected neurodevelopment in only half the studies, indicating that the benefits of consuming mercurycontaining fish might outweigh the negative impact of mercury exposure; ethylmercury did not affect neurodevelopment; hair manganese was a much more consistent biomarker of manganese exposure than blood; and the neurotoxicity of lead was amplified in the presence of manganese.

Conclusions and potential impact: In low and middle-income countries, heavy metals have a significantly detrimental effect on the neurodevelopment of children, highlighting the need for more regulation. 